\title{
Anomalous Scaling in Heteroepitaxial Island Dynamics on Ag(100)
}

\author{
Christopher Zaum, ${ }^{1, *}$ Michael Rieger, ${ }^{2}$ Karsten Reuter, ${ }^{2}$ and Karina Morgenstern ${ }^{1}$ \\ ${ }^{1}$ Institute for Solid State Physics, Division of Atomic and Molecular Structures (ATMOS), Leibniz University of Hannover, \\ Appelstraße 2, D-30167 Hannover, Germany \\ ${ }^{2}$ Fritz-Haber-Institut der Max-Planck-Gesellschaft, Faradayweg 4-6, D-14195 Berlin, Germany
}

(Received 7 February 2011; published 20 July 2011)

Diffusion and decay of alloyed $\mathrm{Cu} / \mathrm{Ag}$ islands are investigated in the size range from 1 to $40 \mathrm{~nm}^{2}$ on $\operatorname{Ag}(100)$ at room temperature with fast-scanning tunneling microscopy and density functional theory. While islands at sizes above $7 \mathrm{~nm}^{2}$ show the diffusion and decay behavior expected for dynamics based on single atom hopping, islands smaller than $4 \mathrm{~nm}^{2}$ diffuse faster and decay slower than predicted by standard theory. This anomalous behavior at unexpected large island sizes is related to a size dependent dealloying of the $\mathrm{Cu} / \mathrm{Ag}$ islands.

The physical properties of nanoscale systems can change abruptly with decreasing system size. These characteristics are often used to tune the electronic or optical performance of quantum dots [1] or 3D nanoparticles [2]. On surfaces, the occurrence of such scaling effects was observed in the emergence of preferred heights in thin metal films $[3,4]$. Later it was detected for lateral scaling of quantum dots and adatom islands [5,6]. All effects investigated so far are so-called quantum size effects, which are related to electron confinement. Such confinement occurs when the size of a nanostructure becomes comparable to the electron's de Broglie wavelength. For metals, this wavelength is typically in the range of a few atomic distances, which is still small compared to the size of nanoparticles used for technological applications.

In this Letter, we present a novel type of scaling effect not related to electron confinement and found in nanoscale structures consisting of up to 100 atoms. We investigate diffusion and decay of $\mathrm{Cu}$ and alloyed $\mathrm{Cu} / \mathrm{Ag}$ islands on $\mathrm{Ag}(100)$ by fast-scanning tunneling microscopy (STM) and density functional theory (DFT) and reveal a transition region from 4 to $7 \mathrm{~nm}^{2}$ for this heteroepitaxial metal system. Above $7 \mathrm{~nm}^{2}$ the islands are composed of a $\mathrm{Cu} / \mathrm{Ag}$ alloy, and application of standard theory to the diffusion and decay behavior of these islands indicates that the island dynamics is based on single atom movements. In the transition region, the islands dealloy. Below a size of $4 \mathrm{~nm}^{2}$ the islands consist only of $\mathrm{Cu}$ atoms adsorbed not exclusively in hollow sites of the $\operatorname{Ag}(100)$ surface. The diffusion of the pure $\mathrm{Cu}$ islands is faster and their decay is slower than predicted by standard theory. DFT relates the displacement of the atoms from hollow sites to the strain induced by the large lattice mismatch between copper and silver. These islands show a stronger internal bond and a weaker bond to the surface explaining the anomalous diffusion and decay behavior.

Measurements are performed with a commercial fast-scanning STM (SPECS "STM 150 Aarhus") at and close to room temperature (RT) and with a custom-built low-temperature STM [7] at $5 \mathrm{~K}$. Both setups are housed in an UHV environment (base pressure $\leq 2 \times 10^{-10} \mathrm{mbar}$ ). The fast-scanning STM is equipped with a stabilization algorithm for recording of image sequences (movies).

The $\operatorname{Ag}(100)$ surface is cleaned by standard sputtering and annealing cycles. After cleaning, 0.003-0.13 ML of copper are deposited with deposition rates between 0.001 and $0.01 \mathrm{ML} / \mathrm{min}$ onto the substrate at RT and in a control experiment at $150 \mathrm{~K}$. At RT, the resulting islands are of monatomic height and quadratic shape with an area between 1 and $40 \mathrm{~nm}^{2}$.

DFT calculations are performed using the plane-wave code CASTEP [8] with the generalized gradient approximation Perdew-Burke-Ernzerhof functional [9] to treat electron exchange and correlation. The surfaces are modeled in supercell geometries with three $\operatorname{Ag}(100)$ layer slabs separated by at least $0.6 \mathrm{~nm}$ vacuum above the last atoms of each structure. All atomic positions in the topmost $\mathrm{Ag}(100)$ layer and in the $\mathrm{Cu}$ islands are fully relaxed until residual forces fall below $350 \mathrm{meV} / \mathrm{nm}$. Systematic convergence tests indicate that the binding energy per $\mathrm{Cu}$ atom is converged below $1 \mathrm{meV}$ at these computational settings.

Figure 1 shows STM images of the $\operatorname{Ag}(100)$ surface after copper deposition at RT. The metal islands are approximately $10 \mathrm{~nm}^{2}$ in size. Their dynamics is followed by repetitively scanning the same spot on the sample to create an image sequence. In total 3500 images representing $46 \mathrm{~h}$ of island evolution are analyzed. Following selected islands in time (marked in Fig. 1 with I and II) reveals two processes: the metal islands diffuse and decay simultaneously. For example, the smaller island no. II shows a significantly increased diffusion rate in contrast to the larger island no. I, which stays in close proximity to its starting point (Fig. 1, white arrow). Furthermore, the island decay depends on the island size.

In order to quantify our results we quickly review the mean field theory, which successfully describes island 

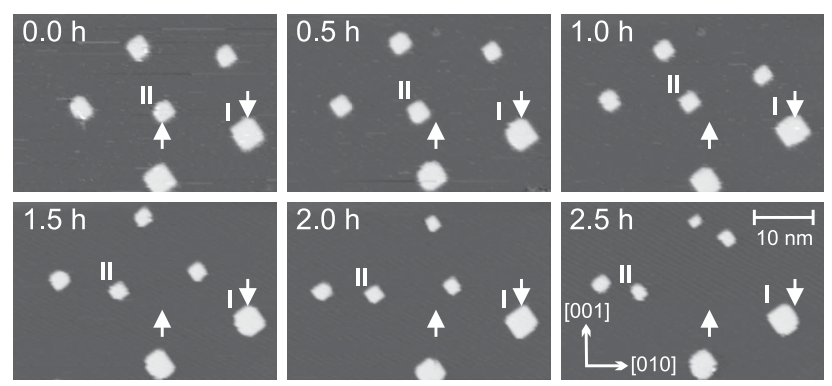

FIG. 1. $\mathrm{Cu}$ islands on $\mathrm{Ag}(100)$. Snapshots of a movie (1771 images, $60 \mathrm{~s} /$ image $)$ at indicated time $\left(I_{t}=790 \mathrm{pA}, V_{t}=\right.$ $610 \mathrm{mV}, T=300 \mathrm{~K})$. Islands I and II are discussed.

decay and diffusion in homoepitaxial systems [10]. The random motion of adatom islands on surfaces can be described as Brownian motion. The probability distribution for finding a moving particle at the distance $\Delta x$ from its starting point after time $\Delta t$ is a Gaussian distribution with its width given by the Einstein relation $\left\langle(\Delta x)^{2}\right\rangle=$ $2 D \Delta t$ [11]. The diffusion constant $D$ is a constant for single adatom diffusion, but it depends on the island size $A$ for adatom island diffusion [12]:

$$
D \propto A^{-\alpha} .
$$

In the case that the island's movement results from independent single atom motion, three limiting mechanisms with different exponents $\alpha$ in Eq. (1) are revealed [13]. For diffusion induced by single atoms moving along the island's periphery [periphery diffusion (PD)], $\alpha$ has a value of 1.5. For islands diffusing by randomly exchanging atoms with the two-dimensional adatom gas on the surface, the diffusion is limited either by the diffusivity of the adatoms on the surface [terrace diffusion (TD)] or by the recondensation process of the adatoms at the island edges [evaporation and condensation limited diffusion (EC)]. Thereby, $\alpha$ is equal to 1 for the TD mechanism and it is equal to 0.5 for the EC mechanism. For the homoepitaxial systems related to this study, the following values for $\alpha$ were reported [14]: $\alpha_{\mathrm{Cu}}=1.25 \pm 0.04$ for $\mathrm{Cu} / \mathrm{Cu}(100)$ islands and $\alpha_{\mathrm{Ag}}=1.14 \pm 0.05$ for $\mathrm{Ag} / \mathrm{Ag}(100)$ islands with island sizes larger than $7 \mathrm{~nm}^{2}$. Monte Carlo simulations suggest $\alpha=1.5$ for $\mathrm{Cu}$ islands on $\mathrm{Cu}(100)$ also for island sizes $A<10 \mathrm{~nm}^{2}$ [15]. This identifies the underlying mechanism as a combination of PD and TD diffusion. For metal islands consisting of only a few atoms, Monte Carlo simulations show a vast increase of $\alpha$ due to the occurrence of concerted motions of the island's atoms [16-18]. In this smaller size range $\alpha$ is no longer a physical quantity.

We now quantify the measured island diffusion at $\mathrm{Ag}$ (100) by extracting the diffusion constant $D$ for islands within different size ranges via the Einstein relation [19]. These diffusion constants are plotted double logarithmically versus the average island size $A$ in Fig. 2(a). Obviously, the data cannot be fitted by a single exponent. Fitting the small and the large islands' diffusivity

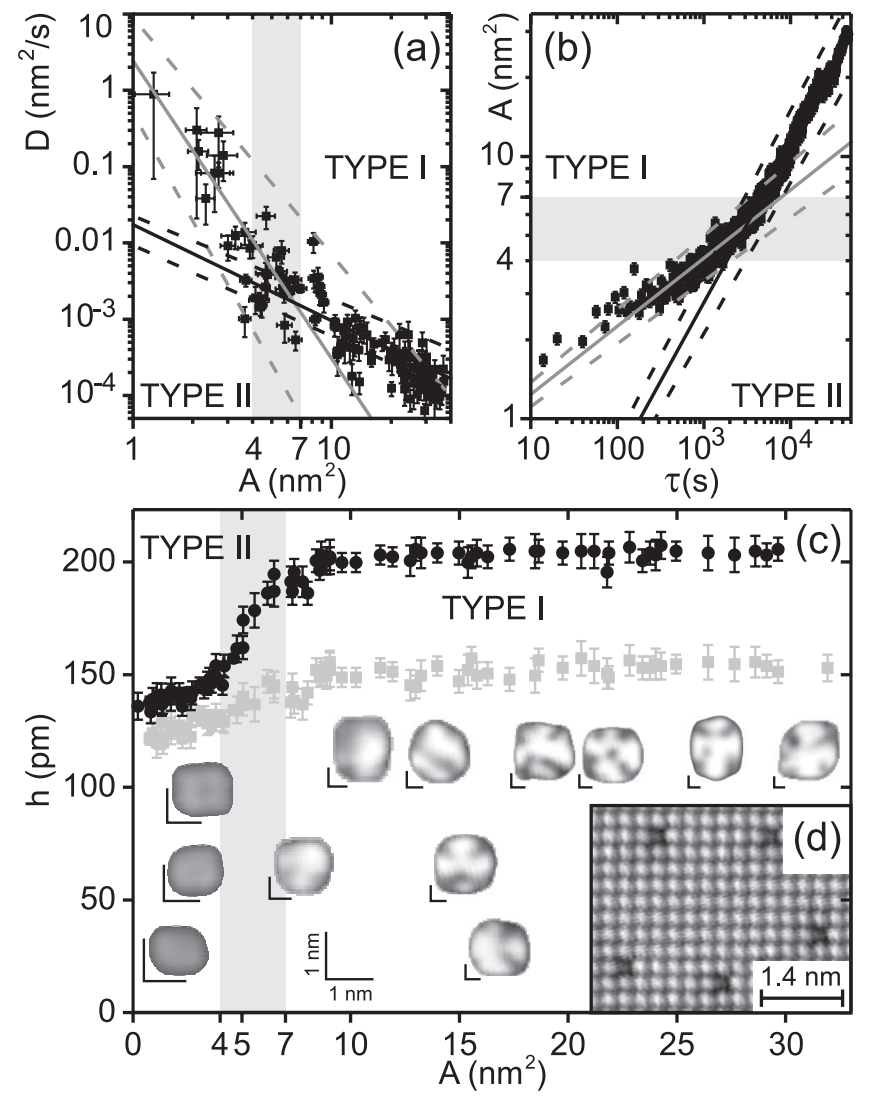

FIG. 2. Island dynamics. (a),(b) Double logarithmic plots of diffusion constant $D$ vs island size $A$ (a) and of island size $A$ vs decay parameter $\tau:=t_{0}-t$ (b) at RT. Solid lines in (a) and (b) show linear fits with error as dashed lines: black lines for islands with $A>7 \mathrm{~nm}^{2} \quad(\alpha=1.26 \pm 0.14, \quad \beta=0.28 \pm 0.01) \quad$ and gray lines for islands with $A<7 \mathrm{~nm}^{2}(\alpha=3.9 \pm 0.8, \beta=$ $0.13 \pm 0.01$ ). (c) Maximum (black dots) and minimum (gray squares) island height $h$ versus island size $A$. Insets: STM images characteristic for the size given on $x$ axis. Note that the STM images are scaled as indicated by the $1 \mathrm{~nm}$ scale bar and that the $z$ scale is 15 times increased as compared to Fig. 1 (all images: $I_{t}=40 \mathrm{pA}, V_{t}=250 \mathrm{mV}, T=5 \mathrm{~K}$ ). (d) Atomic resolution image of $\mathrm{Ag}(100)$ after adsorption of $\mathrm{Cu}$ at $\mathrm{RT}\left(I_{t}=55 \mathrm{pA}\right.$, $\left.V_{t}=425 \mathrm{mV}, T=5 \mathrm{~K}\right)$.

separately by a linear least square fit leads to two different exponents $\alpha$. The two least square fits intersect at a critical island size $A_{\text {crit }}$ in the range of $4<A_{\text {crit }}<7 \mathrm{~nm}^{2}$. For islands with $A>7 \mathrm{~nm}^{2}$ (called type I), we find $\alpha=$ $1.26 \pm 0.14$ [Fig. 2(a), black line]. This agrees well with the result measured for the related systems $\mathrm{Cu} / \mathrm{Cu}(100)$ and $\mathrm{Ag} / \mathrm{Ag}(100)$ [14]. In contrast, we observe $\alpha=3.9 \pm$ 0.8 [Fig. 2(a), gray line] for islands with $A<4 \mathrm{~nm}^{2}$ (called type II). This value for the exponent $\alpha$ is approximately 2 times larger than the values predicted by standard theory. Therefore, the diffusion of type II islands can certainly not be explained by any of the proposed mean field mechanisms.

Proceeding to the decay behavior of the islands, the classical theory of cluster ripening developed by Lifshitz, Sloyozov, and Wagner (LSW) was applied to surfaces by 
Chakraverty, Wynblatt, and Gjostein [20,21]. This theory describes a circular adatom island with a radius $r$ surrounded by a circular step with radius $R$ that acts as a sink for the adatoms. For not too small islands sizes, integration of the stationary diffusion equation leads to a power law for the time evolution of the island's radius $r(t)$ of an island that is completely decayed at $t_{0}$ [10]:

$$
r(t)^{2} \propto A(t) \propto \tau^{2 \beta} \quad \text { with } \tau=\left(t_{0}-t\right) \text { and } \beta=\frac{1}{3} .
$$

This relation was observed for $\mathrm{Ag}$ islands on $\mathrm{Ag}(111)$ [22]. The experimentally obtained value $\beta=0.27 \pm 0.06$ deviates from the calculated value $\beta=\frac{1}{3}$ as expected at small island sizes resulting from the inaccuracy introduced by linearization of the exponential term.

To quantify the island's decay behavior, we renormalize the time scale to the decay parameter $\tau$ [cf. Eq. (2)]. This allows us to display all island decays in a single double logarithmic plot [Fig. 2(b)]. Again these data cannot be fitted by a single exponent $\beta$. As for island diffusion, we also find two types of island decays with a transition between these decay regimes at a critical island size $A_{\text {crit }}$ with $4<A_{\text {crit }}<7 \mathrm{~nm}^{2}$ [Fig. 2(b)]. For type I islands we obtain $\beta=0.28 \pm 0.01$ [Fig. 2(b), black line]. This matches the experimentally obtained value $\beta=$ $0.27 \pm 0.06$ for $\mathrm{Ag}$ islands on $\mathrm{Ag}(111)$ [22]. In contrast, we observe $\beta=0.13 \pm 0.01$ for type II islands [Fig. 2(b), gray line]. This result for $\beta$ is only half of the value predicted by surface adapted LSW theory. So standard theory also does not hold for the decay of small islands.

In the following we will discuss the difference of type I and type II islands, which is at the origin of the different decay and diffusion behaviors, based on high resolution STM and DFT calculations. Imaging the surface prepared at room temperature at $5 \mathrm{~K}$ reveals that depressions $(\Delta h \approx$ $6 \mathrm{pm}$ ) with the size of single adatoms are present on the $\operatorname{Ag}(100)$ terrace [Fig. 2(d)]. Furthermore, the number of observed depressions increases with coverage during RT deposition. A control experiment shows that evaporation of $\mathrm{Cu}$ at $150 \mathrm{~K}$ does not lead to such depressions but to a clean $\mathrm{Ag}(100)$ surface covered with single $\mathrm{Cu}$ adatoms. Only after annealing this surface to $250 \mathrm{~K}$ the depressions and adatom islands are appearing.

These findings provide strong evidence for the formation of a surface alloy. The depressions in Fig. 2(d) are explained by a chemical contrast between $\mathrm{Ag}$ atoms and $\mathrm{Cu}$ atoms alloyed into the $\mathrm{Ag}(100)$ surface. Such a contrast was reported before, e.g., for $\mathrm{Pt}$ on $\mathrm{Rh}(100)$ [23]. After depositing $0.05 \mathrm{ML}$ of copper, $\sim 2 \%$ of the surface atoms are imaged as depressions, indicating that these darker atoms are $\mathrm{Cu}$ atoms. DFT calculations suggest that this alloying results from the exchange diffusion mode for $\mathrm{Cu}$ adatoms on $\operatorname{Ag}(100)$ [24]. Assuming that all $\mathrm{Ag}$ atoms removed from the $\operatorname{Ag}(100)$ surface during alloying are incorporated into the adatom layer leads roughly to a $\sim 1: 1$ alloy ratio for copper and silver.
For islands grown at RT, the two island species deduced from the dynamic analysis differ in their internal structure. For type I islands, regions with two different apparent heights are found within each island with a maximum of $h_{\max } \approx 200 \mathrm{pm}$ [Fig. 2(c), black dots] and a minimum of $h_{\text {min }} \approx 150 \mathrm{pm}$ [Fig. 2(c), gray squares]. Considering that STM measures protruding areas too broad and recessed areas too small, the ratio between higher and lower parts of the type I islands is roughly $1: 1$ and therefore matches the calculated alloy ratio. For type II islands, the corrugation is much smaller $\left(h_{\max } \approx 140 \mathrm{pm}, h_{\min } \approx\right.$ $130 \mathrm{pm}$ ) and no internal island structure is visible in high resolution STM images. The height of the elevated areas in type I islands is close to the step height of the $\operatorname{Ag}(100)$ surface of $204 \mathrm{pm}$. The apparent height of the lower areas of these islands corresponds approximately to the uniform height of type II islands. The transition between structured and uniform islands is likewise situated in the size range from 4 to $7 \mathrm{~nm}^{2}$. We conclude that the formation of the surface alloy during deposition leads to two compositionally different island types. Type I islands are alloyed $\mathrm{Cu} / \mathrm{Ag}$ islands while type II islands are pure $\mathrm{Cu}$ islands.

A possible explanation of the influence of the island's composition on its dynamics results from the adsorption sites of the island atoms. To determine those, we analyze images that simultaneously resolve the islands and the $\operatorname{Ag}(100)$ surface atomically [Figs. 3(a) and 3(b)]. In these images, the grid defined by the $\operatorname{Ag}(100)$ surface is extended to the island. A comparison of the positions of the island atoms to the positions of the $\mathrm{Ag}$ surface atoms reveals that the atoms in elevated areas of type I islands adsorb in
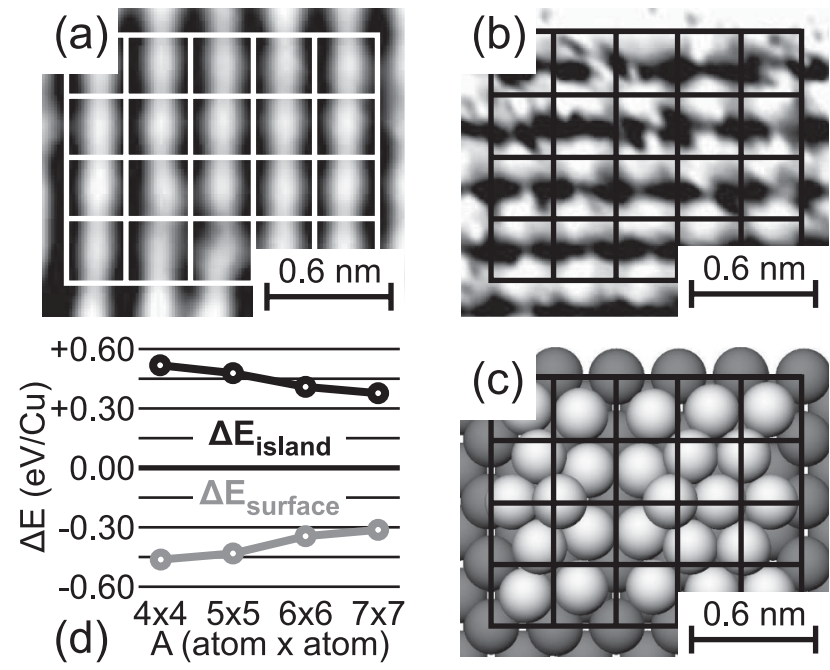

FIG. 3. Island adsorption sites and energies. (a),(b) STM images of island with size (a) $A=33 \mathrm{~nm}^{2}\left(I_{t}=850 \mathrm{pA}, V_{t}=\right.$ $857 \mathrm{mV}, T=220 \mathrm{~K})$ and (b) $A=5.5 \mathrm{~nm}^{2}\left(I_{t}=780 \mathrm{pA}, V_{t}=\right.$ $806 \mathrm{mV}, T=224 \mathrm{~K})$. Grids represent on-top positions of the silver lattice as obtained by extension of the atomically resolved substrate on the same image to the island. (c),(d) Results of DFT calculations. (c) Island with size $A=2.1 \mathrm{~nm}^{2}(5 \times 5)$, (d) stabilization energy $\Delta E$ versus island size $A$. 
hollow sites of the $\operatorname{Ag}(100)$ surface. Atomic resolution of the lower parts of type I islands is much more difficult, but it hints at a deviation from the hollow sites. Even at low temperature the smaller islands of type II are moved at tunneling parameters that allow atomic resolution. In the transition region few islands could be resolved and one of them is shown in Fig. 3(b). In these images the atoms are clearly displaced from hollow sites.

To confirm such a displacement we performed DFT calculations. As expected, adsorption of a monolayer of $\mathrm{Cu}$ into the fourfold coordinated hollow sites of a $\operatorname{Ag}(100)$ surface turns out to be significantly more stable than the adsorption into bridge sites by $-0.434 \mathrm{eV} / \mathrm{Cu}$ atom. However, for square $\mathrm{Cu}$ islands with sizes $(4 \times 4)$ to $(7 \times 7)$ we observe a reconstruction of the entire island structure [e.g., Fig. 3(c)] accompanied by a significant energy gain [e.g., by $+0.083 \mathrm{eV} / \mathrm{Cu}$ atom for the $(4 \times 4)$ island]. In the $(5 \times 5)$ island shown, several $\mathrm{Cu}$ atoms occupy positions close to the nominal bridge sites of the surface consistent with positions revealed in experiment. The reconstruction is driven by the fact that in the reconstructed islands the $\mathrm{Cu}-\mathrm{Cu}$ distances are significantly shortened approaching the $\mathrm{Cu}$ bulk lattice constant.

In order to understand the effect of such a reconstruction onto the islands dynamics, we separated the total stabilization energy $\Delta E=\left(E_{\text {reconst }}-E_{\text {hollow }}\right) / N_{\text {atoms }}$ for the different island structures into a bonding contribution $\Delta E_{\text {surface }}$ that arises from the bonding to the $\operatorname{Ag}(100)$ substrate and a bonding contribution $\Delta E_{\text {island }}$ arising from the inner island $\mathrm{Cu}-\mathrm{Cu}$ bonding [Fig. 3(d)]. $\Delta E_{\text {island }}$ decreases and $\Delta E_{\text {surface }}$ increases with increasing island size. This provides a natural rationalization for an enhanced mobility of the smaller reconstructed $\mathrm{Cu}$ islands compared to the larger unreconstructed alloy islands. In the former, the main stabilization comes from strong intraisland $\mathrm{Cu}-\mathrm{Cu}$ bonds with a concomitantly less important $\mathrm{Cu}$-substrate interaction, whereas in the latter the main stabilization comes from the $\mathrm{Cu}-\mathrm{Ag}$ interaction itself. Therefore, the reconstructed islands are more prone to diffusion, simply because the average island-substrate interaction is smaller than in the case of the unreconstructed islands. Even the probability of a collective diffusion process that would involve more than a single $\mathrm{Cu}$ island atom at once is higher for a more compact island structure than for an island in which the island atoms are less bound amongst each other. Molecular dynamics simulations suggest that such a rapid diffusion by gliding motion is feasible for the related heteroepitaxial system $\mathrm{Ag} / \mathrm{Cu}(100)$ [25]. On the same footing, it is possible to understand the anomalous decay exponent. The inner island bonding of the reconstructed type II islands is higher than for the unreconstructed ones and therefore these islands are less likely to decay. The definition of the diffusion exponent $\alpha$ and the decay exponent $\beta$ relies on a constant adatom diffusion barrier. Because the internal island bonding and the island-surface bonding vary strongly with the island's size, the anomalous large exponents for type II island diffusion and the anomalous small exponents for their decay are no longer physical quantities.

In conclusion, two metal island species with different dynamic behavior are formed in the $\mathrm{Ag} / \mathrm{Cu}(100)$ system. The islands with normal dynamic attributes (type I) consist of a $\mathrm{Cu} / \mathrm{Ag}$ alloy and dealloy to pure $\mathrm{Cu}$ islands (type II) in a size range from 7 to $4 \mathrm{~nm}^{2}$. A size dependent shift in intraisland versus island-surface bonding causes an anomalous decay and diffusion with decreasing island size. The obtained large values for the diffusion exponent $\alpha$ suggest a novel diffusion mechanism not described by standard theory. This diffusion and decay behavior as well as the size dependent dealloying is likely to occur also in other heteroepitaxial systems with large lattice misfits as often used in industrial applications. From a scientific point of view, these processes belong to a novel class of nanoscale size effects beyond those caused by confined electrons at much smaller sizes.

We acknowledge financial support by the DFG.

*zaum@fkp.uni-hannover.de

[1] S. M. Reimann and M. Manninen, Rev. Mod. Phys. 74, 1283 (2002).

[2] M. Pelton, J. Aizpurua, and G. W. Bryant, Laser Photon. Rev. 2, 136 (2008).

[3] B. Hinch et al., Europhys. Lett. 10, 341 (1989).

[4] R. Otero, A. L. Vázquez de Parga, and R. Miranda, Phys. Rev. B 66, 115401 (2002).

[5] S. Pons, P. Mallet, and J.-Y. Veuillen, Phys. Rev. B 64, 193408 (2001).

[6] K. Morgenstern, E. Lægsgaard, and F. Besenbacher, Phys. Rev. Lett. 94, 166104 (2005).

[7] M. Mehlhorn et al., Rev. Sci. Instrum. 78, 033905 (2007).

[8] S. J. Clark et al., Z. Kristallogr. 220, 567 (2005).

[9] J. P. Perdew, K. Burke, and M. Ernzerhof, Phys. Rev. Lett. 77, 3865 (1996).

[10] K. Morgenstern, Phys. Status Solidi B 242, 773 (2005).

[11] A. Einstein, Ann. Phys. (Leipzig) 322, 549 (1905).

[12] A. F. Voter, Phys. Rev. B 34, 6819 (1986).

[13] S. V. Khare and T.L. Einstein, Phys. Rev. B 54, 11752 (1996).

[14] W. W. Pai et al., Phys. Rev. Lett. 79, 3210 (1997).

[15] M. O. Jahma et al., Surf. Sci. 598, 246 (2005).

[16] O. S. Trushin et al., Surf. Sci. 482-485, 365 (2001).

[17] P. Salo et al., Phys. Rev. B 64, 161405 (2001).

[18] A. Karim et al., Phys. Rev. B 73, 165411 (2006).

[19] Class width is $\Delta A=1 \mathrm{~nm}^{2}$ for $A>4 \mathrm{~nm}^{2}$ and $\Delta A=$ $0.5 \mathrm{~nm}^{2}$ for $A \leq 4 \mathrm{~nm}^{2}$.

[20] B. K. Chakraverty, J. Phys. Chem. Solids 28, 2401 (1967).

[21] P. Wynblatt and N. A. Gjostein, Prog. Solid State Chem. 9, 21 (1975).

[22] K. Morgenstern, G. Rosenfeld, and G. Comsa, Phys. Rev. Lett. 76, 2113 (1996).

[23] E. L. D. Hebenstreit et al., Surf. Sci. 441, 441 (1999).

[24] H. Yildirim and T. S. Rahman, Phys. Rev. B 80, 235413 (2009).

[25] O. U. Uche et al., Phys. Rev. Lett. 103, 046101 (2009). 